Ebisu

Études japonaises

Études japonaises

47 | printemps-été 2012

Catastrophes du 11 mars 2011, désastre de

Fukushima : fractures et émergences

\title{
Fermiers sinistrés de Fukushima : comment faire face dans des conditions extrêmes?
}

The Effects of March 11th on the Farm Families of Fukushima: How to Face Up to Extreme Conditions?

極限の選択を生る福島の被次農民たち

\section{Hiroko Humbert-Amemiya}

\section{(2) OpenEdition \\ 1 Journals}

Édition électronique

URL : http://journals.openedition.org/ebisu/401

DOI : 10.4000/ebisu.401

ISSN : 2189-1893

Éditeur :

Institut français de recherche sur le Japon (UMIFRE 19 MAEE-CNRS), Maison franco-japonaise

\section{Édition imprimée}

Date de publication : 1 juin 2012

Pagination : 165-172

ISSN : $1340-3656$

\section{Référence électronique}

Hiroko Humbert-Amemiya, «Fermiers sinistrés de Fukushima : comment faire face dans des conditions extrêmes ? », Ebisu [En ligne], 47 | printemps-été 2012, mis en ligne le 03 avril 2014 consulté le 18 septembre 2019. URL : http://journals.openedition.org/ebisu/401 ; DOI : 10.4000/ ebisu.401 


\title{
Fermiers sinistrés de Fukushima Comment faire face dans des conditions extrêmes ?
}

\author{
Hiroko HUMBERT-AMEMIYA アンベール-雨宮 裕子
}

«Mon mari s'est tué pour protester contre Tepco ${ }^{1}$ ». C'est ainsi que s'est exprimée publiquement Mme Tarukawa Mitsuyo 樽川美津代. L'émotion remplissait la salle qui écoutait le récit de la veuve. Touché par son témoignage, Nemoto Satoshi 根本敬, secrétaire général de la Nōminren 農民連 ${ }^{2}$ (Fédération nationale de paysans) du département de Fukushima a réagi en décidant de se présenter à une élection locale pour établir la responsabilité de Tepco et du gouvernement. Sugeno Seiju 菅野正寿, agriculteur biologique présent également à la réunion, a décidé de rester vivre à Fukushima ${ }^{3}$

\footnotetext{
- Maître de conférences, université de Rennes 2, directrice du Centre de recherches sur la culture japonaise de Rennes, mène des recherches au Japon sur les relations villecampagne depuis 2008.

1. Tepco est le terme utilisé pour désigner de manière abrégée l'entreprise d'électricité Tokyo Electric Power Company (Tōkyō denryoku kabushiki gaisha 東京電力株式会社).

2. Abréviation de Nōmin undō zenkoku rengōkai 農民運動全国連合会. Cette Fédération nationale des Paysans a été fondée en 1989 pour défendre l'agriculture paysanne familiale et compte 50000 membres fermiers dans l'ensemble du Japon. Depuis 2008, elle est affiliée à Via Campesina, le mouvement international pour la défense de l'agriculture durable de petite échelle. Les agriculteurs japonais sont en principe tous membres de la coopérative nationale des agriculteurs, la JA (Japan Agricultural Cooperatives, Nōkyō 農協). La constitution de la Nōminren s'explique par un mécontentement des petits fermiers qui ne se sentaient pas bien soutenus par la politique de la JA.
}

3. Ceux dont la ferme est dans une zone de $20 \mathrm{~km}$ de la centrale, interdite pour une période encore indéfinie, ne pourraient faire ce choix même s'ils le souhaitaient. 
en continuant à pratiquer une agriculture biologique et montrer ainsi que l'on peut retrouver «son beau pays».

Face à l'épreuve de la vie et de la mort, comment les paysans sinistrés de Fukushima vont-ils projeter leur avenir ? En m'engageant dans des actions pour les soutenir, j'ai beaucoup échangé avec eux. Nombreux sont ceux qui vivent encore dans les tourments de l'indécision sous la pression des liens de parenté et des communautés. Mais certains paysans ont déjà choisi leur chemin. À partir de quelques exemples rencontrés, nous pouvons essayer de comprendre de quelle manière ils cherchent à organiser leur avenir, et à se reconstruire tout en affichant leur identité.

\section{Le premier suicide protestataire d'un agriculteur biologique}

Le 26 avril 2011, une délégation de fonctionnaires du ministère de l'Agriculture, des Forêts et de la Pêche (Nōrin suisan shō 農林水産省) avait accepté une rencontre semi-publique, à laquelle j'ai participé, au ministère, avec le groupe de paysans de la Nōminren venu réclamer une indemnisation rapide 4 . Sur ce dernier point, les fonctionnaires les ont renvoyés vers Tepco, la compagnie d'électricité. Les agriculteurs ont alors fait part de leur désarroi, car ils revenaient d'une rencontre au siège de Tepco qui ne cherchait qu'à retarder la procédure. Comment survivre d'ici là sans avoir reçu la moindre indemnité alors que nombre d'entre eux étaient déjà au bout du rouleau?

Un paysan dans la salle a dit : "Sauver la vie du peuple, c'est le rôle du gouvernement. Pourquoi le nôtre ne veut pas s'engager ? " Les fonctionnaires ont juste promis de faire de leur mieux pour achever l'entretien.

La réunion s'est poursuivie avec Mme Tarukawa Mitsuyo. Son mari, âgé de 64 ans, agriculteur maraîcher biologique à Sukagawa 須賀川 depuis 35 ans s'est suicidé le 29 mars. Mme Tarukawa a raconté son entretien

4. Sur les demandes d'indemnisation portées par la Nōmiren, voir l'article de Namioka Shintarō " Politiques de dédommagement pour les agriculteurs de Fukushima ? Le cas de la Nōminren " dans le présent numéro [N.D.L.R]. 
du matin avec des responsables de Tepco. Nous avons écouté son propos timide mais clair :

"Mon mari s'est suicidé pour protester contre Tepco. Il était encore en forme après le tremblement de terre. Il allait secourir les blessés. Il a été touché par les images de l'accident de la centrale et il disait : "Pourquoi le Japon qui a connu la bombe atomique a-t-il adopté l'énergie nucléaire ?” [...] J'ai des petits-enfants. Nous portons toujours un masque et nous ne pouvons étaler ni lessive ni futons dehors, à l'air libre. C'est dur. On se pose des questions. Peut-on vendre ce que nous cultivons ? $\mathrm{Si}$ je démarre les concombres, seront-ils achetés ? Je continue à cultiver parce que je deviendrai folle si je restais à la maison sans rien faire. Pourvu que l'accident soit mâ̂trisé le plus vite possible. Je cultive le riz mais je me demande si je pourrai le vendre, et si je n’empoisonne pas mes clients avec du riz contaminé par la radioactivité. On m’a dit qu'il suffit de bien le laver..."

\section{Le combat de Nemoto Satoshi}

Nemoto Satoshi (55 ans) a décidé de se présenter aux élections du conseil général du département de Fukushima, dans la circonscription de Nihonmatsu 二本松 (à $50 \mathrm{~km}$ de la centrale, mais avec une radioactivité significative). Il y avait deux postes à pourvoir et il était le troisième candidat. Pour Nemoto Satoshi, c'était un défi car à la campagne on a le plus souvent une préparation qui permet d'avoir autant de candidats que de postes à pourvoir. Il se présentait sans étiquette mais avait reçu le soutien du Parti communiste. L'élection n'était pas facile à organiser, nombre d'électeurs étant dispersés, réfugiés loin de la centrale.

Bien qu'il ait su le trouble créé auprès des électeurs par sa campagne tardive, celle-ci n'était nullement hésitante car c'était le début de son combat pour " retrouver un Fukushima, beau et prospère ${ }^{5}$ » qui représente le souhait le plus cher de tous. Il dit dans sa déclaration de candidature :

" On parle de la reconstruction. Pour reconstruire, il faut commencer par les hommes. Pour que nous puissions vivre humainement, nous devons nous battre.

5. Nemoto emploie cette expression : «yutaka de utsukushii Fukushima» 豊かで美しい 福島 (un Fukushima beau et prospère) comme tous ceux qui luttent pour la renaissance de Fukushima. 
Pour qu'il n'y ait plus jamais des lieux sinistrés ni des personnes sinistrées par le nucléaire, nous devons nous battre. Moi, je poursuis mon combat jusqu'à ce que je puisse transmettre le beau Fukushima et non pas le Fukushima souillé à mes enfants et à mes petits enfants. "

Il a été touché par la retenue de Mme Tarukawa Mitsuyo lors de la première rencontre avec les responsables de Tepco. Tenant la photo de son mari qui s'est suicidé, elle n'a pas blâmé les employés de Tepco. Elle a juste demandé d'arrêter la centrale au plus tôt en pensant aux enfants. Cependant, Nemoto Satoshi, lui, pose des questions : "Qui a souillé Fukushima par la radioactivité ? Qui a rendu la vie si difficile aux femmes et aux enfants comme disait Mitsuyo ? " Tepco exige des sinistrés qu’ils apportent la preuve des dégâts dont ils souffrent pour accepter de les dédommager. Les paysans ne sont pas responsables de l'accident. Ils sont des victimes. Mais leur droit à la vie n'est pas assuré s'ils ne le revendiquent pas.

Il a déclaré : "Je vais consacrer toute ma vie de paysan pour retrouver un Fukushima beau et prospère ». Désormais sa vie sera un combat pour sortir du nucléaire et pour défendre la vie et l'agriculture du Japon ${ }^{6}$.

\section{Cultiver pour transmette une terre fertile aux générations futures}

Sugeno Seiju (53 ans) a pris une autre orientation. Il s'efforce de nettoyer sa terre pour pouvoir la transmettre aux générations suivantes. Il fournit tous les efforts possibles pour continuer à vivre où il est, parce que "la fuite " entrânerait la destruction de la communauté et que sa terre fertile biologique serait également perdue. Né à Nihonmatsu, il pratique l'agriculture biologique depuis 30 ans sur 2,5 ha de rizière et 1 ha de terre maraîchère. Il est le représentant à Fukushima du Réseau national de l'agriculture biologique du Japon ${ }^{7}$ et il s'investissait dans le développement de sa communauté autour de l'agriculture.

6. Le journal Nōmin 農民 (Paysans), le 7 novembre 2011. Il n’a pas été élu cette fois-ci. Il était troisième, avec deux mille voix, derrière le second élu.

7. Fukushima-ken yūki nōgyō nettowāku 福島県有機農業ネットワーク. 
Pour Sugeno Seiju, il n'y a pas à choisir d'aller vivre ailleurs. Touché par le témoignage de Mme Tarukawa Mitsuyo, il ne voulait plus voir d'autres suicides parmi ses collègues alors que ceux-ci se succédaient. En juin, un éleveur de la ville de Sōma 相馬 s'est pendu en laissant le mot suivant : "J'ai perdu courage. Pourvu que les autres éleveurs résistent malgré la centrale ${ }^{8}$ ". Une dame de 93 ans s'est suicidée chez elle en laissant un petit mot : "Je vais me réfugier dans le tombeau ${ }^{9}$ ". Dans le département de Fukushima, il y a eu 160 suicides entre les mois d'avril et de juin, soit $20 \%$ de plus qu'en 2010 sur la même période ${ }^{10}$.

Voyant l'augmentation du nombre de suicides, Sugeno Seiju a décidé de vivre d'une façon positive " comme une fleur de tournesol qui s'épanouit dans les champs en luttant contre la radioactivité ${ }^{11}$ ". Le tournesol et le colza sont, en effet, les plantes conseillées pour enlever la radioactivité du sol et Sugeno Seiju a suivi l'idée pour en expérimenter l'efficacité. Mais avant même l'examen du sol, il a eu le grand plaisir de voir le tournesol fleurir. Il se sentait encouragé par la vitalité de cette fleur qui dressait la tête malgré la contamination. En tant qu'agriculteur biologique, il continue à prendre soin de sa terre. Il examine minutieusement le sol à divers endroits de sa propriété pour surveiller le taux de radioactivité et il rend le résultat public pour maintenir la transparence. Ses champs sont devenus un laboratoire pour tester les techniques avancées de décontamination. Sa bataille livrera des données précieuses, son histoire également, celle d'un agriculteur biologique qui se veut "modèle ». Il a conclu une intervention en disant qu'il allait consacrer toute son énergie à la transmission du « beau Fukushima " aux générations futures.

8. Journal Yomiuri 読売新聞, 14 juin 2011.

9. Journal Mainichi 毎日新聞, 9 juillet 2011 : <http://mainichi.jp/select/weathernews/20110311/news/20110709k0000m040149000c.html> (consulté le 9 juillet 2011).

10. Journal Mainichi, 9 juillet 2011 : <http://mainichi.jp/select/weathernews/ news/20110709k0000m040151000c.html> (consulté le 9 juillet 2011).

11. Cité d'après son intervention le 5 novembre à Kyoto au cours du symposium : "Junkan kanō na enerugī o umidasu yūki nōgyō shinpojiumu » 循環可能なエネルギー を生みだす有機農業シンポジウム (Pour une agriculture biologique créatrice d'énergie renouvelable), organisé par Nō o kaetai ! Zenkoku undō kansai chiiki nettowāku 農を 変えたい! 全国運動関西地域ネットワーク (Réseau de la région du Kansai du mouvement national «Changeons l'agriculture!»). 
Sugeno Seiju est un homme solide et ingénieux. Il serait un bon leader pour une communauté nouvelle constituée de paysans sinistrés et de paysans locaux, dans un endroit non contaminé et relativement déserté comme il y en a beaucoup au Japon ; constituer de telles communautés est une des propositions pour lesquelles nous avions cherché des soutiens. Je lui ai demandé franchement s'il partirait ailleurs pour reconstruire sa vie si on lui proposait la même surface cultivable et les mêmes conditions de vie. "Bien sûr que non ", m'a-t-il répondu d'un ton ferme. "Je n'ai aucune intention de partir. Il n'y a pas de raison de partir. Si tout le monde partait, notre communauté n'existerait plus. »

\section{Cultiver dans un environnement sain pour protéger la santé et en priorité celle des enfants}

«C'est ma femme qui a soutenu l'idée du départ » relate Tanno Kisaburō 丹 野喜三郎 (70 ans), qui pratiquait sur 60 a de rizières et 2 ha de champs une agriculture biologique depuis 40 ans à Nihonmatsu. Midori 緑, sa femme, pour protéger ses enfants et son petit fils, s'est montrée résolue à quitter définitivement et à mettre les jeunes hors de danger. Leur ferme n'était pas dans une zone officiellement contaminée, et leur départ les empêche de solliciter tout type d'indemnités (pour mévente par exemple). Mais le couple Tanno, convaincu de la nécessité de pratiquer une agriculture saine pour des aliments sains, avait dans cette conviction une raison de plus de partir. En effet, bien que hors zone contaminée, leurs épinards ont été refusés en mars 2011 par la coopérative de consommateurs Seikyō 生協 ${ }^{12}$ en raison d'un taux trop élevé d'éléments radioactifs. Ils avaient cultivé des petits pois sur une superficie de $1000 \mathrm{~m}^{2}$ mais ils ont décidé de les jeter car la coopérative Seikyō ne prendrait plus aucun légume provenant de chez eux. C'est à ce moment-là qu'ils ont eu la visite de personnes de l'Association pour la recherche sur l'agriculture biologique ${ }^{13}$ dont Tanno Kisaburō était membre, et qui lui ont demandé s'il souhaitait s'installer ailleurs. Elles ont parlé des possibilités d'accueil dans les départements de Nagano et de

12. Abréviation de Nihon seikatsu kyōdō kumiai rengōkai 日本生活協同組合連合会.

13. Nihon yūki nōgyō kenkyūkai 日本有機農業研究会. 
Yamanashi. Le couple Tanno a choisi Ueda 上田 dans le département de Nagano où ils se sont installés avec leurs enfants dès la fin du mois de mai. Là, comme en beaucoup d'endroits, les terres abandonnées ne manquent pas à cause du vieillissement des agriculteurs sans successeurs. La famille Tanno a trouvé une ferme à louer à Bessho onsen 別所温泉, un hameau de la ville d'Ueda ${ }^{14}$.

Tanno Kisaburō a reçu une offre de location de champs d'une superficie de 3 ha et un collègue du hameau de Saku 佐久 lui a prêté une rizière. Grâce à l'altitude élevée et au climat tempéré, on peut cultiver toutes sortes de légumes. Les terrains abandonnés se trouvent plutôt en hauteur et sous forme de petites parcelles. Le père s'intéresse d'abord à la qualité de la terre alors que son fils aîné, également maraîcher biologique, veut trouver un grand terrain plat pour qu'il puisse utiliser un tracteur. Entre père et fils, bien qu'ils pratiquent tous les deux l'agriculture biologique, l'accord n'est pas immédiat quant à la gestion des activités. Le fils aîné est plus inquiet pour l'avenir de la famille dans un nouvel environnement sans soutien financier de l'État.

Le 9 juillet, la soirée d'accueil de la famille Tanno organisée par la NPO locale "Shoku to nō no machizukuri nettowāku » 食と農の町づくりネット ワーク (Réseau pour revigorer la communauté locale par les aliments et l'agriculture) ${ }^{15}$ était animée par la participation de plusieurs couples de jeunes néo-ruraux, installés ou préparant leur installation. Kisaburō a été longuement interrogé par ces jeunes sur les techniques agricoles et il était particulièrement heureux de leur donner des conseils fondés sur sa longue expérience.

14. La ville d'Ueda compte 160000 habitants et se trouve à 1 h 20 en train rapide Shinkansen du centre de Tokyo.

15. Créée en 2009, l'association composée de citoyens locaux et d'universitaires a monté, après le désastre du 11 mars, un projet pour soutenir les paysans sinistrés de Fukushima : elle leur propose un hébergement et des terres agricoles disponibles afin de faciliter leur départ des zones hautement radioactives et de se réinstaller dans la région d'Ueda. 


\section{Pour conclure}

L'agriculteur fait un métier indissociable de la terre, source de sa vie et de ses activités. La catastrophe nucléaire de Fukushima a mis les paysans, surtout ceux de la zone d'évacuation, dans une situation difficile à surmonter. Dispersés dans des centres de refuge ou des hébergements précaires, ils sont en situation de survie. S'ils veulent continuer une vie de paysan, il leur faut accepter d'aller s'installer dans un lieu inconnu, loin de leurs fermes, héritages familiaux de plusieurs générations. S'ils n'arrivent pas à s'aventurer, ils deviendront dépendants de l'aide sociale comme chômeur. Chaque famille doit trouver une solution le plus rapidement possible en réunissant les informations et en cherchant les bonnes opportunités. Ces choix dans des conditions extrêmes, bien que contraints, laissent apparaître un aspect positif : cela peut être une occasion que n'auraient jamais eu les paysans de décider de prendre en main leur vie de manière autonome, de leur propre initiative. Libérés d'une charge ancestrale, ils peuvent choisir leur avenir et leur cadre de vie. C'est comme si le désastre nucléaire avait rompu les liens qui les avaient ancrés depuis si longtemps dans la terre natale.

De fait, quelques-uns ont déjà choisi leur chemin. Partir, ou rester, ou encore poursuivre sa vie autrement que dans les activités agricoles. Quel que soit le choix, leurs liens affectifs à la terre natale survivront à jamais dans le « beau Fukushima » qu'ils croient avoir connu dans le passé. Embelli dans leur imagination, ce « beau Fukushima » est devenu le symbole de leur combat éternel. 